\title{
Gute wissenschaftliche Praxis im Öffentlichen Recht
}

Die Mitglieder der Vereinigung der deutschen Staatsrechtslehrer verstehen die folgenden Leitsätze guter wissenschaftlicher Praxis als Selbstvergewisserung über die Grundlagen ihrer Arbeit in ihrem Teilgebiet der Jurisprudenz. Sie gehen nicht davon aus, dass sich die Arbeit im Öffentlichen Recht von der in anderen juristischen Fächern substantiell unterscheidet, halten es aber gleichwohl für sinnvoll, disziplinär die eigene Sicht darzulegen, weil das Gespräch über gute wissenschaftliche Praxis in der Jurisprudenz auf den in den Fächern gesammelten Erfahrungen aufbauen muss.

Die Leitsätze wollen als die Arbeit begleitende „Regeln der Kunst“ gelesen werden, die das wissenschaftsadäquate Verhalten beschreiben, und nicht als Verbandsregelwerk mit gesetzesgleicher Kraft. Sie erheben auch nicht den Anspruch, die autonomen Normgeber in den Fakultäten zu verpflichten. Wo Organisationsvorstellungen die Leitsätze grundieren, orientieren sie sich an häufig vorfindlichen Strukturen, die aber selbstverständlich auch anders gestaltet sein können, ohne den Zweck der Regel auf den es ankommt - zu gefährden.

I.

Plagiate, Autorschaft, Zitierstandards

1. Wissenschaftsplagiate, d.h. die vollständige oder teilweise Übernahme eines fremden Textes oder einer fremden Idee unter Anmaßung der wissenschaftlichen Urheberschaft, verstoßen gegen die Pflicht zur Wahrhaftigkeit der Wissenschaft.

2. Gleichermaßen wissenschaftlich unredlich ist die Veröffentlichung eines von Anderen verfassten Textes unter eigenem Namen mit deren Einverständnis - entgeltlich oder unentgeltlich („Ghostwriting“).

3. Es ist wissenschaftlich unredlich, wenn ein Hochschullehrer Textentwürfe durch seine Mitarbeiter fertigen lässt und diese unter eigenem Namen als Alleinautor veröffentlicht.

4. Eine Mitwirkung, die in ihrer intellektuellen Leistung für einen Beitrag wesentlich ist, führt zur (Mit-)Autorschaft.

5. Eine bloß sprachliche Überarbeitung lässt die Autorschaft des Entwurfsverfassers nicht entfallen. $\mathrm{Ob}$ die Hochschullehrerin oder der 
Hochschullehrer überhaupt eine Autorschaft für sich beanspruchen kann, hängt davon $a b$, ob sie oder er qualitativ oder quantitativ Wesentliches zum Entwurf beigesteuert hat.

6. Die unterstützende Mitwirkung wissenschaftlicher Mitarbeiterinnen und Mitarbeiter begründet nur dann keine Autorschaft, wenn die Mitwirkung sich in bloßen Hilfstätigkeiten wie Recherche, Materialsammlung, Arbeit an den Fußnoten und vergleichbaren Routinen erschöpft. Dafür kann in einer Fußnote gedankt werden.

7. Bei gemeinsamer Veröffentlichung mehrerer Autoren soll soweit möglich zukünftig ausgewiesen werden, welche Leistung die jeweilige Autorschaft begründet.

8. In wissenschaftlichen Veröffentlichungen muss der Leser erkennen können, inwieweit der Autor sich jenseits des Allgemeinkundigen auf Ergebnisse und Formulierungen Dritter stützt.

9. Wörtliche Übernahmen eines fremden Textes bzw. Textteils sind durch Zitatzeichen oder, insbesondere bei Übernahme längerer Textpassagen, in anderer geeigneter Form (z.B. eingerückter Text in abweichender Schriftart) zu kennzeichnen.

10. Bei der wörtlichen oder sinngemäßen Übernahme eines fremden Textes bzw. Textteils ist die Quelle (Autor/-in und Fundstelle) durch die Platzierung oder Gestaltung der Fußnote so präzise anzugeben, dass sie überprüft werden kann. Es genügt nicht, die wissenschaftliche Literatur lediglich in einer "Sammelfußnote“ oder in einem Literaturverzeichnis aufzuführen. Bei einem Zitat sind den Zitierten keine Aussagen zuzuschreiben, die diese nicht oder nicht in der angegebenen Weise gemacht haben.

11. Im Grundsatz entspricht es wissenschaftlicher Redlichkeit, primär den Urheber einer Idee zu zitieren; Sekundärquellen werden deshalb nur neben und nach Primärquellen aufgeführt.

12. „Blindzitate“, d.h. die ungeprüfte Übernahme der Zitate Anderer, verstoßen grundsätzlich gegen die Standards der Wissenschaft. Wenn Quellen nicht mit zumutbarem Aufwand überprüft werden können, erfolgt ein entsprechender Hinweis (etwa „zitiert nach ...").

13. Allgemeinwissen im Sinne von Grundwissen, dessen Kenntnis im Fach vorausgesetzt werden kann, ist nicht zitierbedürftig. Insbesondere Wissen, das Allgemeingut geworden ist, muss dem Urheber oder der Urheberin nicht mehr zugeordnet werden. Zitierbedürftig ist die Wiedergabe von Allgemeinwissen nur, wenn eine vorfindliche besondere Formulierung Ausdruck einer urheberrechtlich schützenswerten persönlichen geistigen Schöpfung ist.

14. Die Auswahl von Zitaten ist ausschließlich anhand wissenschaftsadäquater Kriterien vorzunehmen. Für die Darstellung des Standes der 
Wissenschaft des öffentlichen Rechts verbietet die Zitierredlichkeit wissenschaftsfremde Kriterien für die Auswahlentscheidung anzuwenden, etwa solche ,wissenschaftspolitischer" oder persönlicher Art (z.B. Freundschaft, Verbundenheit in Wissenschaftszirkeln) und so „Zitierkartelle" zu bilden.

15. Abweichungen von den vorgenannten Zitierstandards können mit Blick auf die zweck- und zielgruppenadäquate Gestaltung einer Publikation gerechtfertigt sein (z.B. bei einem Beitrag in einer Zeitung). Der Grundsatz, dass die Übernahme fremden Gedankenguts offen gelegt werden muss, gilt allerdings auch hier.

II.

Gute wissenschaftliche Praxis zur Plagiatsvermeidung bei wissenschaftlichen Qualifikationsschriften

16. Es gehört in Prüfungsverfahren für wissenschaftliche Qualifikationsarbeiten ebenso wie im fachwissenschaftlichen Diskurs zur guten wissenschaftlichen Praxis, durch die Ausgestaltung der organisatorischen Rahmenbedingungen zur Vermeidung von Plagiaten beizutragen.

17. Die Betreuer wissenschaftlicher Arbeiten wirken als Mentoren im Rahmen der Verfahren zur wissenschaftlichen Qualifizierung nach ihrem Ermessen darauf hin, dass bestehende Anreize zum Plagiieren soweit wie möglich wirkungslos bleiben.

18. Die Betreuer begründen in den Gutachten für die Prüfungsverfahren, welche Maßnahmen sie für angemessen gehalten und durchgeführt haben.

19. Wissenschaftliche Arbeiten sind im Rahmen von Prüfungsverfahren auch als elektronische Datei einzureichen, um sie leichter mit vorhandenen Datenbeständen abgleichen zu können. Es bleibt den Betreuern, Prüfern oder anderen Mitgliedern der Prüfungs- oder Promotionsausschüsse im Verfahren überlassen, aus welchem Anlass und in welchem Umfang sie die entsprechenden Überprüfungen vornehmen.

20. Das vielfach praktizierte „Patentrezept“ einer eidesstattlichen Versicherung über eine täuschungsfreie, die Regeln guter wissenschaftlicher Praxis beachtende Erarbeitung der Dissertation bei Einreichung der Arbeit ist weniger empfehlenswert, weil ihre Leistungsfähigkeit gemeinhin überschätzt und die damit verbundenen strafrechtlichen Probleme unterschätzt werden.

21. Es entspricht guter wissenschaftlicher Praxis, auch jenseits von Prüfungsverfahren im wissenschaftlichen Diskurs auf Text- und Ideendiebstahl aufmerksam zu machen, statt ihn zu beschweigen. 
22. Bei Prüfungsarbeiten ist eine Zweit- oder Mehrfachverwertung nicht ausgeschlossen, wenn sie offengelegt wird und nach der Prüfungsordnung rechtlich zulässig ist.

23. Im Umfang beschränkte Seminararbeiten dürfen zu Diplom- oder Bachelor-/Masterarbeiten ausgeweitet werden, diese im Zuge von Dissertationen berücksichtigt und dabei zweit- oder gar drittverwertet werden, soweit die neue Studie in ihrer Gesamtheit quantitativ und qualitativ einen eigengearteten Neuigkeitswert von Gewicht aufweist.

\section{III.}

\section{Promotionsverfahren}

\section{Annahme}

24. Promotionsverhältnisse werden mit der Fakultät dadurch begründet, dass ein Hochschullehrer einen Bewerber dem Promotionsausschuss als Doktoranden vorschlägt. Der Promotionsausschuss prüft die Voraussetzungen der Zulassung.

25. Die Zugangsvoraussetzungen sind normativ so $\mathrm{zu}$ fassen, dass ein hohes Leistungsvermögen der Doktoranden vorausgesetzt wird. Dieses wird durch qualifizierte Examensleistungen und zusätzlich durch während des Studiums erbrachte hervorragende Seminarleistungen oder durch ein, einer hervorragenden Seminarleistung entsprechendes, Exposé zu dem zu bearbeitenden Thema nachgewiesen.

26. Doktoranden können ihre Dissertation in einem Beschäftigungsverhältnis mit der Universität, in einem Stipendienverhältnis oder auch extern erbringen. Die nebenberuflich erarbeiteten Dissertationen ermöglichen es, Wissenspotentiale der Praxis für das Öffentliche Recht fruchtbar zu machen. Sie sind nicht täuschungsanfälliger oder qualitativ minderwertiger als im Näheverhältnis zur Universität entstehende Arbeiten, wenn allen Doktoranden die Regeln guter wissenschaftlicher Arbeit vermittelt werden.

27. Graduiertenschulen mit einem Promotionsstudium sind ein geeignetes Format zur Förderung des Promovierens. Aber auch die für eine hermeneutische Wissenschaft typische Individualbetreuung bleibt möglich - auch um die intrinsischen Ressourcen eines Betreuungsverhältnisses zu nutzen.

28. Bereits für etwaige Probleme in der Phase der Begründung eines Doktorandenverhältnisses sollte die Fakultät eine Vertrauensperson bestellen, die mit Rat und ggf. Vermittlung helfen kann. 


\section{Betreuung}

29. Die Betreuung von Doktorandinnen und Doktoranden und von Habilitandinnen und Habilitanden ist zeitlich und inhaltlich anspruchsvoll. Betreuung beginnt u.a. mit der Unterstützung bei der Auswahl und Eingrenzung des Promotions- bzw. Habilitationsthemas und setzt stete Gesprächsbereitschaft voraus.

30. Der Betreuer oder die Betreuerin begleitet den Arbeitsfortschritt durch das Angebot von Statusbesprechungen, die Hinweise zum Fortschritt der Qualifikationsarbeit beinhalten. Ein weiteres Mittel der Betreuung außerhalb von Graduiertenschulen ist ein Doktorandenseminar, das zur Rechenschaft über den Stand der Arbeit zwingt.

31. Wenn der Doktorand seine sich aus dem Promotionsverhältnis ergebenden Pflichten nicht erfüllt, ist der Betreuer berechtigt, das Doktorandenverhältnis zu beenden.

32. Betreuung darf von der Intensität her die für eine selbständige Leistung notwendige Distanz zwischen Betreuer und Doktorand nicht unzulässig verkürzen. Die Autorschaft muss trotz Betreuung beim Doktoranden verbleiben.

33. Betreuung ist zeitbeanspruchende Arbeit im Ensemble der Hochschullehrerpflichten. Deshalb muss die Zahl der Doktoranden, für die der Betreuer oder Betreuerin Verantwortung übernimmt, notwendig limitiert sein. Als Orientierungsgröße ist an etwa zehn gleichzeitige Individualbetreuungsverhältnisse zu denken.

\section{Prüfungsphase}

34. Für die Erstellung der Voten ist eine Regelfrist von je drei Monaten angemessen.

35. Der Promotionsausschuss bestimmt die Gutachter; als Erstgutachter bestellt er regelmäßig den Betreuer, soweit die Promotionsordnung Betreuung und Begutachtung nicht trennt.

36. Der Zweitgutachter wird so ausgewählt, dass sich nicht ohne sachlichen Grund regelmäßige Berichterstatter-Paare bilden, um der Besorgnis möglicher Unsachlichkeit vorzubeugen.

37. Lässt sich eine hinreichende Gutachter-Rotation innerhalb einer Fakultät personell nicht verwirklichen, werden Zweitgutachten auswärtig vergeben. Das gilt vor allem, wenn es um Spezialthemen geht, für die es in der Fakultät keinen zweiten mit der Problematik hinreichend vertrauten Gutachter gibt.

38. Obligatorische auswärtige Drittgutachten, etwa für mit summa cum laude bewertete Arbeiten, schwächen die Profilbildung der Fakultät; abgesehen davon ist zweifelhaft, ob die Drittgutachten die in sie 
gesetzten Erwartungen hinsichtlich einer Qualitätskontrolle erfüllen können.

39. Dissertationsvoten erschöpfen sich nicht in einer bloßen Plausibilitätskontrolle. Beide Voten müssen jene Wissensvermehrung in der Dissertation präzise benennen, die die Annahme der Arbeit rechtfertigen; die bewertungsmäßige Verbindung des Grades des Wissensfortschritts ist im Abgleich mit dem Stand der Forschung zu der vorgeschlagenen Note darzulegen. In die Bewertung wird weiter der Grad der Vollständigkeit der Materialverwertung, die Verständlichkeit der Gedankenführung und deren sprachliches Niveau einbezogen. Der Vorschlag der Höchstnote muss die hervorragende Bedeutung der Dissertation begründen.

40. Das mündliche Examen sollte so konzipiert sein, dass die intellektuelle Urheberschaft des Doktoranden für die Arbeit durch die Art der Fragestellung mitgeprüft wird.

\section{IV. \\ Förderung des wissenschaftlichen Nachwuchses}

41. Es gehört zu den Aufgaben der Hochschullehrerinnen und Hochschullehrer, sich gezielt um die Förderung des wissenschaftlichen Nachwuchses zu bemühen. Dazu kann z.B. der Abschluss von (schriftlichen) Betreuungsvereinbarungen mit Doktorandinnen und Doktoranden und Habilitandinnen und Habilitanden beitragen.

42. Dem wissenschaftlichen Nachwuchs wird ausreichend Gelegenheit auch über das gesetzlich angeordnete Maß hinaus - zu eigener Forschung und hochschuldidaktischer Qualifikation gegeben. Insbesondere ist hinreichend Zeit für die Arbeit an den Qualifikationsschriften zu geben.

43. Habilitandinnen und Habilitanden erhalten auf eigenen Wunsch Gelegenheit, selbständig Vorlesungen zu halten.

44. In der Zusammenarbeit von Hochschullehrerinnen und Hochschullehrern und wissenschaftlichem Nachwuchs sind dessen autorschaftliche Ansprüche zu respektieren und eine selbständige Publikationstätigkeit zu fördern.

45. Es ist geboten, den wissenschaftlichen Nachwuchs von Anfang an in wissenschaftsethischen Fragen zu schulen. Die hier formulierten Standards guter wissenschaftlicher Praxis müssen dem wissenschaftlichen Nachwuchs auch vorgelebt werden. 


\section{V. \\ Transparenz}

46. Staatsrechtslehrerinnen und Staatsrechtslehrer erstatten u.a. Staatsorganen und Körperschaften Gutachten. Wenn daraus, was durchaus geboten ist, Publikationen hervorgehen, ist die Beziehung zu einem Auftraggeber in einer Fußnote offenzulegen. Das gilt auch für Folgeveröffentlichungen in Orientierung an solchen Rechtsgutachten. Entsprechendes gilt, wenn ein Wissenschaftler an einem Prozess als Bevollmächtigter beteiligt gewesen ist und $\mathrm{zu}$ dem Prozessthema publiziert.

47. Interessenkonflikte, die die Besorgnis der Befangenheit begründen können, sind offenzulegen. Das gilt insbesondere für Begutachtungen in wissenschaftsbezogenen Antragsverfahren, Peer-Review-Verfahren, Evaluationsverfahren und Berufungsverfahren, aber auch bei einer finanziellen Förderung eines Forschungsvorhabens und seiner Veröffentlichung durch Dritte.

48. Es ist schlechte wissenschaftliche Praxis, eigene veröffentlichte Texte später im Wesentlichen unverändert ohne Nachweis auf ihre ursprüngliche Fundstelle zu publizieren, so dass der Anschein einer Erstpublikation entsteht.

49. Öffentlich-rechtliche Zeitschriften mit wissenschaftlichem Anspruch sichern einen hohen Qualitätsstandard. Sie legen offen, in welchem Verfahren und nach welchen Kriterien über die Annahme von Beiträgen entschieden wird.

50. Über die Annahme von Beiträgen wird ausschließlich nach wissenschaftsadäquaten Kriterien entschieden; Peer-Review-Verfahren sind dazu ebenso geeignet wie kollegiale Herausgeberentscheidungen.

In der Fassung der Beschlüsse der Mitgliederversammlung am 3. Oktober 2012 in Kiel. 
